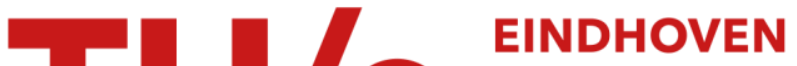 UNIVERSITY OF TECHNOLOGY
}

\section{Data fusion of electrophysiological and haemodynamic signals for ventricular rhythm tracking}

\section{Citation for published version (APA):}

Thoraval, L., Carrault, G., Schleich, J. M., Summers, R., Velde, van de, M., \& Diaz, J. (1997). Data fusion of electrophysiological and haemodynamic signals for ventricular rhythm tracking. IEEE Engineering in Medicine and Biology Magazine, 16(6), 48-55. https://doi.org/10.1109/51.637117

DOI:

10.1109/51.637117

Document status and date:

Published: 01/01/1997

\section{Document Version:}

Publisher's PDF, also known as Version of Record (includes final page, issue and volume numbers)

\section{Please check the document version of this publication:}

- A submitted manuscript is the version of the article upon submission and before peer-review. There can be important differences between the submitted version and the official published version of record. People interested in the research are advised to contact the author for the final version of the publication, or visit the $\mathrm{DOI}$ to the publisher's website.

- The final author version and the galley proof are versions of the publication after peer review.

- The final published version features the final layout of the paper including the volume, issue and page numbers.

Link to publication

\section{General rights}

Copyright and moral rights for the publications made accessible in the public portal are retained by the authors and/or other copyright owners and it is a condition of accessing publications that users recognise and abide by the legal requirements associated with these rights.

- Users may download and print one copy of any publication from the public portal for the purpose of private study or research.

- You may not further distribute the material or use it for any profit-making activity or commercial gain

- You may freely distribute the URL identifying the publication in the public portal.

If the publication is distributed under the terms of Article 25fa of the Dutch Copyright Act, indicated by the "Taverne" license above, please follow below link for the End User Agreement:

www.tue.nl/taverne

Take down policy

If you believe that this document breaches copyright please contact us at:

openaccess@tue.nl

providing details and we will investigate your claim. 


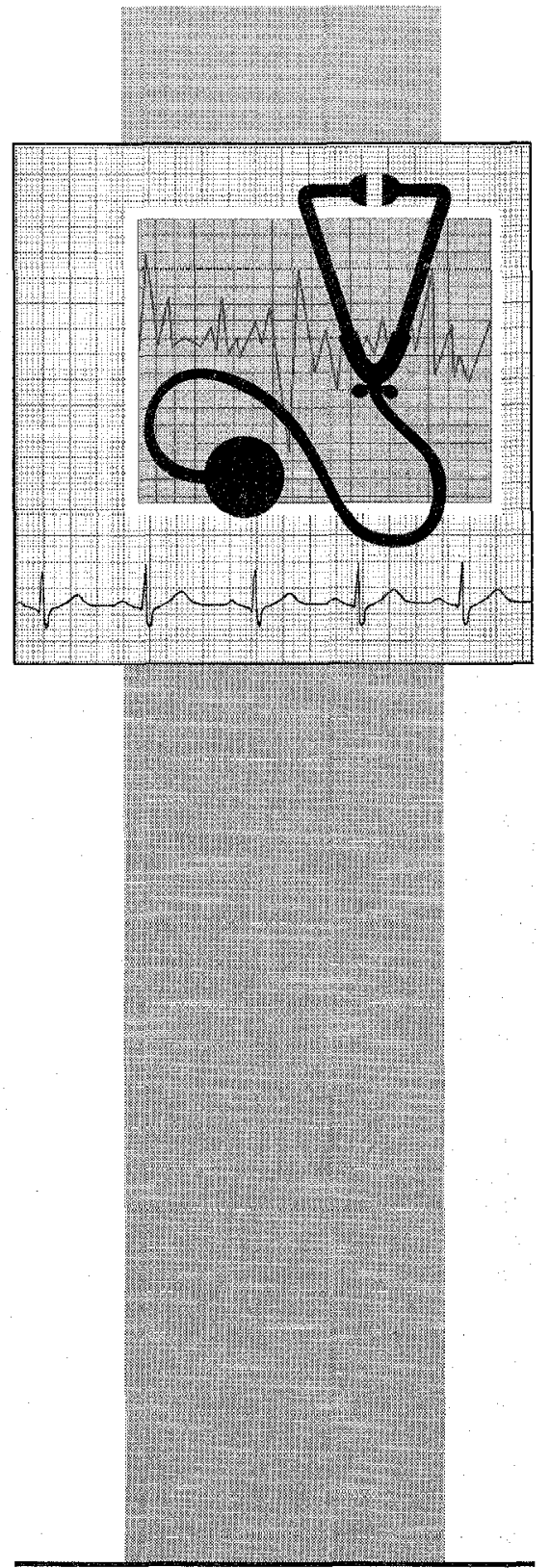

L. Thoraval', G. Carrault', J.M. Schleich ${ }^{2}$ R. Summers, M. Van de Velde ${ }^{4}$ J. Diar ${ }^{5}$ 'LISI University of Rennes I, Beoulieu Campus ${ }^{2}$ CHRU Rennes, Service de cardiologie B, Remnes, Fronce ${ }^{3}$ Cin University, London tEindhoven University of Technology

Data Fusion of Electrophysiological and Haemodynamic Signals for Ventricular Rhythm Tracking

\section{Preliminary Results Based on the IMPROVE Data Library}

$0^{\text {nit }}$ ne of the functions of patient monitoring systems used in critical-care units (CCUs) is to detect, characterize, and automatically generate alarms for each potential life-threatening event. One important physiological signal to track is the ventricular rhythm (VR) $[1,2]$. VR is presently tracked either by observation of the electrocardiogram (ECG) or by following the haemodynamic response, typically by observing a pressure channel [3]. This is especially true of the monitoring of VR in the large number of patients admitted to CCUs after proven or suspected myocardial infarction (MI) or with severe myocardial ischemia.

Disturbances of VR in this setting may relate to rate, rhythm, or the morphology of the ventricular complexes within the electrocardiograph (ECG) trace. These events are common in this setting and may be associated with life-threatening complications or sudden cardiac death. Early detection and prompt treatment of these potentially lethal events along the lines agreed upon by the European Resuscitation Council is the very essence of "coronary care." There is no reason to doubt that this discipline can be extrapolated to other chinical situations where a patient may be at risk of myocardial ischemia or MI.

Although VR tracking seems a relatively simple monitoring task, the signal lacks robustness and its efficiency remains limited despite great research efforts during the last two decades [4-6]. Several reasons can explain this limitation; among these are:

- the composite nature of the noise (stationary and transient) superimposed within the signal(s) that the VR is derived from,
- the low level of signal-to-noise ratio in a high "noise" environment of the $\mathrm{CCU}$, and

- sensor failures, which lead to false alarms and missed detections.

This article aims at demonstrating how the cause of the ventricular contraction (VC), reflected by its electrophysiological precursors (ECG), and its related effect on the haemodynamic system (here characterized by the systemic arterial pressure (SAP), sometimes called the mean systolic arterial pressure, (MSAP)) can be fused together for a VR tracking purpose. Data fusion of heterogeneous signals, such as ECG and SAP, appears to be an interesting alternative to overcome the relative drawbacks of signal processing on univariate channels. Behind the clinical aim of providing a more robust VR signal, this article exemplifies how to manage such heterogeneous data when asynchronous events represent the same $\mathrm{VC}$.

From an engineering perspective, the main objectives of this article are first to present a formal representation of the problcm of VR tracking and, secondly, to introduce a novel signal-processing architecture that is able to manage and fuse cardiovascular data that are both asynchronous and heterogeneous. The evaluation of the performance of this signal-processing system uses a very noisy data set extracted from the IMPROVE data library (DL) [7].

\section{Problem Statement}

Let us suppose we observe the twodimensional random process, $X(k)=\left[X_{1}(k)\right.$ $\left.X_{2}(k)\right]^{T}$, where $k$ denotes the discrete time index, while subscripts 1 and 2 refer, respectively, to the ECG and SAP channels: 


$$
\begin{aligned}
& X_{1}(k)=\sum_{i=1}^{N V} E_{i}\left(k-t_{i}\right)+B_{1}(k) \\
& X_{2}(k)=\sum_{j=1}^{N V} S_{j}\left(k-t_{j}^{\prime}\right)+B_{2}(k)
\end{aligned}
$$

$E_{i}(k)$ and $S_{j}(k)$ are assumed to be realizations of the $E$ and $S$ stochastic processes, respectively, with expectations $E(k)$ and $S(k)$ such that:

$$
\begin{aligned}
& \operatorname{Pr}\left(E(k) \neq 0 \mid k<0, k>K_{E}-1\right)=0 \\
& \operatorname{Pr}\left(S(k) \neq 0 \mid k<0, k>K_{S}-1\right)=0
\end{aligned}
$$

Hence,

$$
\boldsymbol{E}_{i}=\left\{E_{i}\left(t_{i}\right), \ldots, E_{i}\left(t_{i}+K_{E}-1\right)\right\}
$$

and

$$
\boldsymbol{S}_{j}=\left\{S_{j}\left(t_{j}^{\prime}\right), \ldots, S_{j}\left(t_{j}^{\prime}+K_{s}-1\right)\right\}
$$

can be both considered as short-term signals, occurring, respectively, at times $t_{i}$; and $t_{j}^{\prime} . \boldsymbol{E}_{i}$ and $S_{j=i}$ correspond, respectively, to the ECG and SAP components of the same ith VC. For the other terms, $\boldsymbol{E}_{i}$ represents the $i$ th $\mathrm{QRS}$ complex while $S_{i}$ represents the $i$ th SAP systolic pressure wave. Although the time series, $T S_{1}=\left\{t_{i}\right\}$ and $T S_{2}=\left\{t_{i}^{\prime}\right\}$, that describe the true time occurrences of $\left\{\boldsymbol{E}_{i}\right\}_{1 \leq i \leq N V}$ and $\left\{\boldsymbol{S}_{i}\right\}_{1 \leq i \leq N V}$ are asynchro-

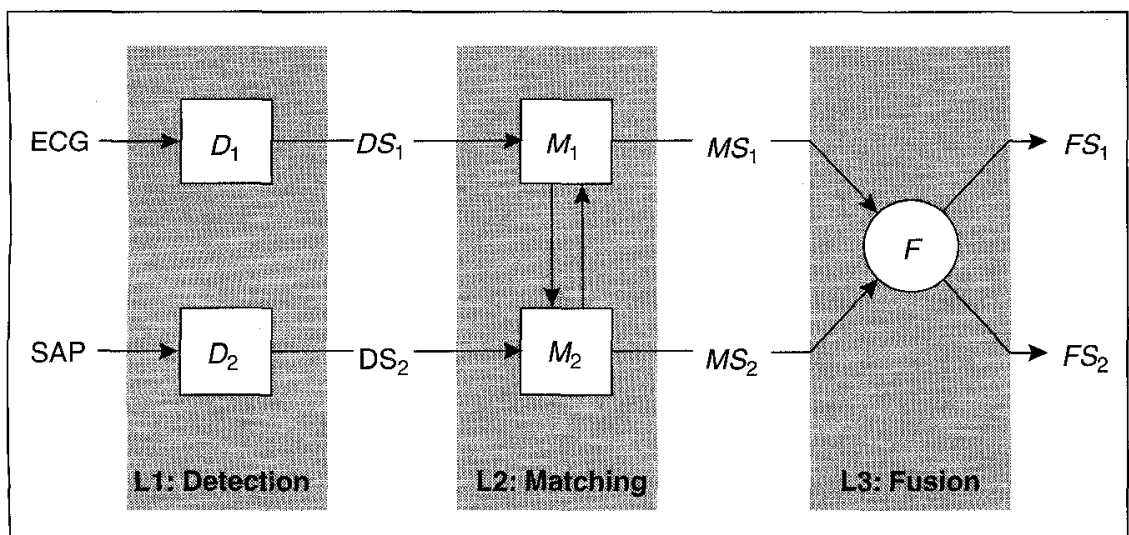

1. Synoptic diagram of the proposed VC detection structure based on ECG and SAP data fusion.

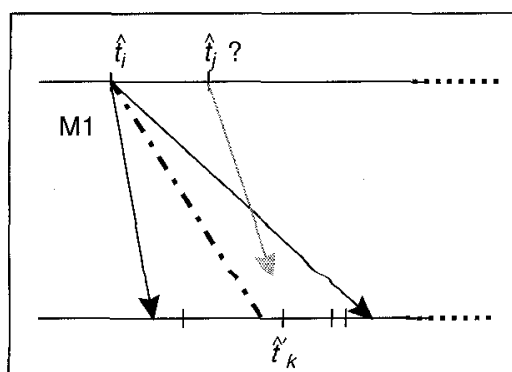

(a)

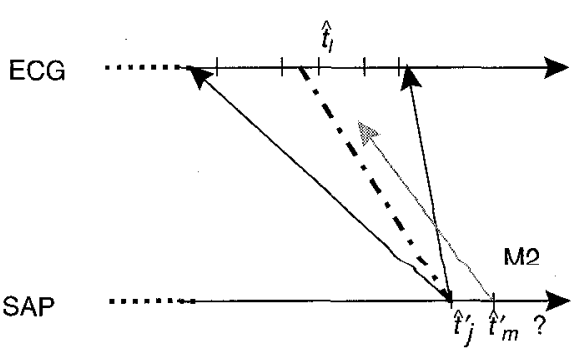

(b)
2. Pictorial description of the matching procedures: a) $M_{1}$, and b) $M_{2}$ (see text).

nous, they are strongly correlated, since the time series $\Delta=\left\{\Delta_{i}=t_{i}-t_{i}^{\prime}\right\}$ here is related to a physiological representation of the electromechanical delay.

$B_{1}(k)$ and $B_{2}(k)$ represent noisy activities observed on the ECG and SAP channels, respectively. They do not correspond to any ventricular activity of interest, namely the QRS complexes and the systolic pressure waves to be detected, but they can corrupt them and even completely mask their observation. Throughout this article, no further hypothesis is assumed about the properties of $B_{1}(k)$ and $B_{2}(k)$. (To illustrate the difficulties in managing noisy situations, examples of representative situations to be processed are depicted in Fig. 3.)

The problem addressed in this article comprises the correct labeling of all NV-successive VC. By formulating the problem statement in this way, we propose to track the VR from data that are not only multichannel but also multimodal. We are aware of approaches that use multichannel/monomodal data, e.g., multilead surface ECGs or vectorcardiograms (VCGs), in the case of work by Gritzali [5]. 
$M S_{1}$ and $M S_{2}$. These latter events are finally merged into two output sequences of time-stamped labels, $F S_{1}$ and $F S_{2}$, by a data-fusion node, $F$. This fusion center does not fuse only symbolic information, that is, the presence or absence of a ventricular event, but also numeric information related to the time occurrences of the ECG and SAP components of each estimated $\mathrm{VC}$.

\section{Level 1: Detection}

Level 1 aims to estimate the true time series, $T S_{1}$ and $T S_{2}$, from the noisy activities, $B_{1}(k)$ and $B_{2}(k)$. Estimating $T S_{1}$ or $T S_{2}$ is usually solved by constructing a decision statistic that enhances the waves, $\left\{E_{i}\right\}_{1 \leq i \leq N Y}$ or $\left\{S_{j}\right\}_{1 \leq j \leq N Y}$, of interest, while minimising the influence of the corresponding noisy activities, $B_{1}(k)$ or $B_{2}(k)$. The statistic is then thresholded to derive the detected time sequence, either
$D S_{1}=\left\{\left(E, \hat{t}_{i}\right)\right\}$ or $D S_{2}=\left\{\left(S, \hat{t}_{j}^{\prime}\right)\right\}$, wherein the labels $E$ and $S$ are added to fulfill the requirements of the subsequent levels L1 and L2.

Among QRS complex detectors proposed during the last two decades, two have been retained to be tested and compared in estimating the $T S_{1}$ and $T S_{2}$ series. The first, denoted $D_{1}{ }^{G R}$, has been proposed recently by Gritzali [5] as a generalization of several other QRS complex detectors. The second, denoted $D_{1}{ }^{P T}$, is derived from the QRS complex detector of Pan and Tompkins [15], which is usually considered as one of the most efficient QRS complex detectors for noisy situations. Its input bandpass filter has been redesigned to take into account the $100 \mathrm{~Hz}$ sampling frequency of the ECG signal as well as 50 $\mathrm{Hz}$ power-line interference.
SAP, as other blood-pressure signals, can be roughly considered as a serratelike signal, wherein discontinuities coincide with systolic ejection time points, so that a simple derivative filter should be well suited to detect these arrival times. As a consequence, the length transformation proposed by Gritzali was retained.

\section{Level 2: Matching}

Matching aims to enhance the likelihood of each outcoming time-stamped label from the previous level by searching for a suitable match on the opposite channel with a time-stamped label that may correspond to the same VC. This match is performed starting from either channel by testing for the electromechanical delay between match candidates. As described in Fig. 2(a), the matching procedure, $M_{1}$, proceeds from channel 1 according to the following steps:

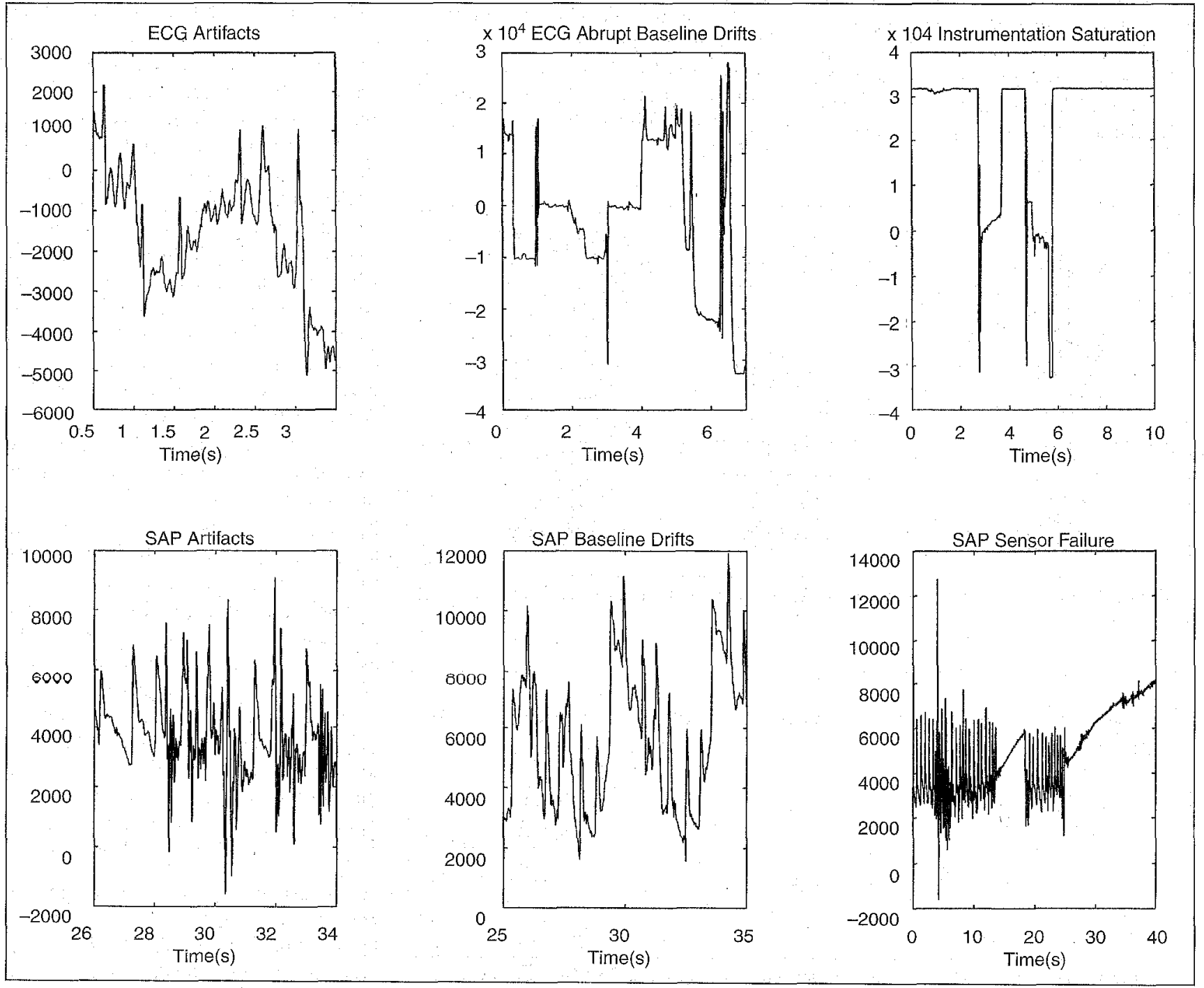

3. Examples of noisy situations managed from the subset of the IMPROVE data library. 


\begin{tabular}{|c|c|c|}
\hline \multicolumn{3}{|c|}{$\begin{array}{l}\text { Table 1. Definition of the fusion rules } \\
\text { used for a fusion node F } \\
\text { acting as a logical operator AND. }\end{array}$} \\
\hline \multicolumn{3}{|c|}{$F \equiv A N D$} \\
\hline Input & \multicolumn{2}{|c|}{ Output } \\
\hline$M S_{1} / M S_{2}$ & $F S_{1}$ & $F S_{2}$ \\
\hline$\left(E, \hat{t}_{i}, S, \hat{t}_{j}\right)$ & $\left(E, \hat{t}_{i}\right)$ & $\left(S, \hat{t}_{j}\right)$ \\
\hline$\left(E, \hat{t_{i}}, \varnothing, 0\right)$ & - & - \\
\hline$\left(\varnothing, 0, S, \hat{t}_{j}\right)$ & - & - \\
\hline
\end{tabular}

The search-window parameter, $\sigma$, and the estimated mean electromechanical delay, $\hat{\bar{\Delta}}$, are considered fixed parameters. Their magnitudes will be defined later. At step $3 \mathrm{a}, \varnothing$ and $\hat{t}_{k}^{\prime}=0$ will indicate to the subsequent fusion node, $F$, that the time-stamped label $\left(E, \hat{t}_{i}\right)$ remains un-

From each time-stamped label $\left(E, \hat{t}_{i}\right) \in D S_{1}$ :

1) open, on channel 2 , a $2 \sigma$ wide search window $W_{\rightarrow 2}=\left[\hat{t}_{i}+\hat{\bar{\Delta}}-\sigma, \hat{t}_{i}+\hat{\bar{\Delta}}+\sigma\right]$,

2) within $W_{1,2}$, search for a match with $\left(S, \hat{t}_{k}^{\prime}\right) \in D S_{2} \quad$ such that $k=\underset{j}{\operatorname{ArgMin}}\left|\hat{t}_{i}-\hat{t}_{j}^{\prime}+\hat{\bar{\Delta}}\right|$

3) If $k$ does not exist, append the 4tuple $\left(E, \hat{t}_{i}, \varnothing, 0\right)$ in $M S_{1}$ and go to step 7

4) otherwise, search for a better match, $\left(E, \hat{t}_{j}, S, \hat{t}_{k}^{\prime}\right)$, such that $\hat{t}_{i}<\hat{t}_{j} \leq \hat{t}_{i}+2 \sigma$

5) if $j$ does not exist, append the 4-tuple $\left(E, \hat{t}_{i}, S, \hat{t}_{k}^{\prime}\right)$ in $M S_{1}$ and go to step 7

6) otherwise, append the 4-tuple $\left(E, \hat{t_{i}}, \varnothing, 0\right)$ in $M S_{1}$

7) $i=i+1$, return to step 1

must be point time-stamped label, $\left(S, \hat{t}_{k}^{\prime}\right) \in D S_{2}$, once matched, cannot be reassigned by $M_{1}$.

The matching $M_{2}$ procedure proceeds similarly on channel 2, as depicted in Fig. 2(b). From $\left(S, \hat{t}_{j}^{\prime}\right) \in D S_{2}$, the $2 \sigma$ wide search window

is opened on channel 1 , within which the best time-stamped label, $\left(E, \hat{t}_{l}\right) \in D S_{1}$, is searched so that:

$$
l=\underset{i}{\operatorname{ArgMin}}\left|\hat{t}_{j}^{\prime}-\hat{t}_{i}-\hat{\bar{\Delta}}\right|
$$

In case of no match candidate in $W_{2 \rightarrow 1}$, the 4-tuple $\left(\varnothing, 0, S, \hat{t}_{j}^{\prime}\right)$ is arbitrarily pro-

$$
W_{1 \rightarrow 2}=\left[\hat{t}_{j}^{\prime}-\hat{\bar{\Delta}}-\sigma, \hat{t}_{j}-\hat{\bar{\Delta}}+\sigma\right]
$$

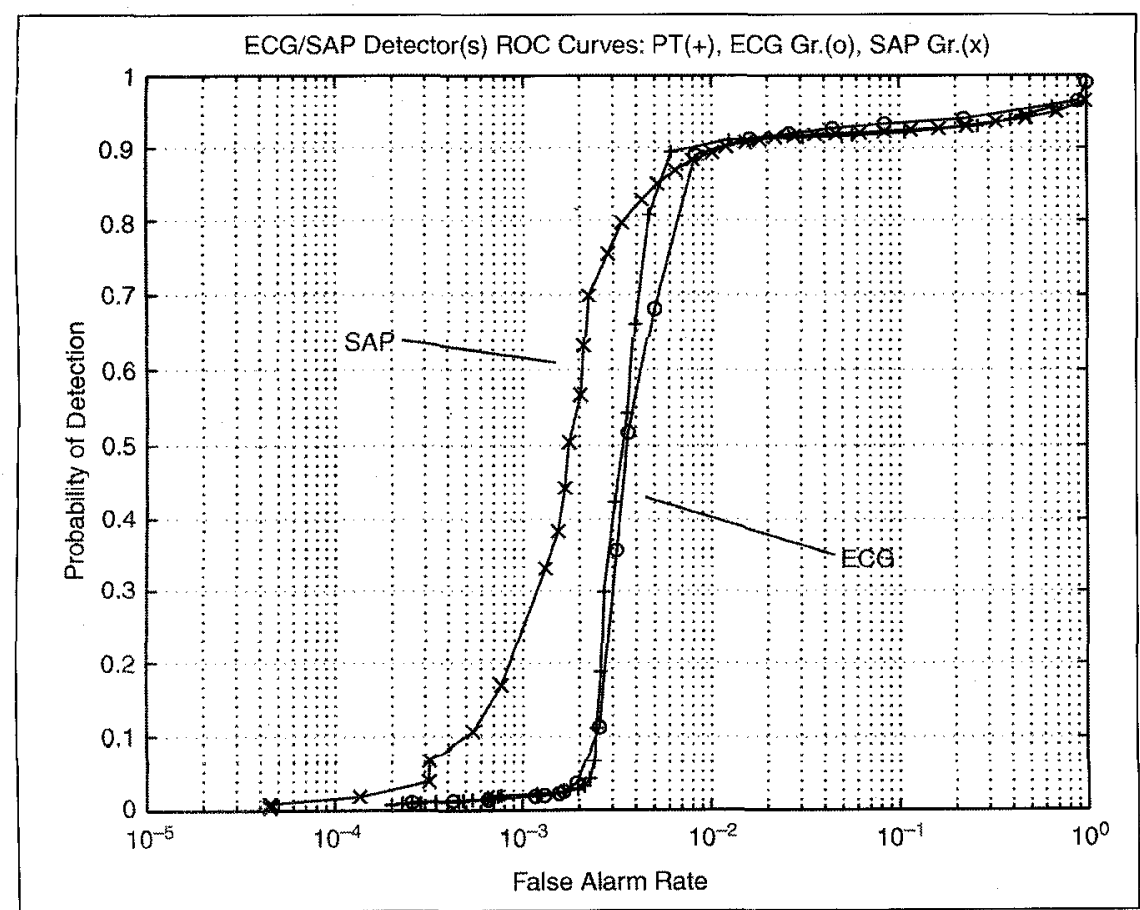

4. VC detection ROC curves obtained at the output of Level 1 (detection), for the three detectors used, namely, $D_{1}^{G R}, D_{1}^{P T}$, and $D_{2}^{G R}$.

\section{The proposed}

approach can easily be

$$
\text { extended to the }
$$

processing of an

$N$-dimensional input

signal.

duced in $M S_{2}$. Otherwise, the 4-tuple $\left(E, \hat{t}_{l}, S, \hat{t}_{j}^{\prime}\right)$ is temporarily created in $M S_{2}$, and then validated, unless a better match candidate, $\left(E, \hat{t}_{l}, S, \hat{t}_{m}^{\prime}\right)$, is detected such that $\hat{t}_{j}^{\prime}<\hat{t}_{m}^{\prime} \leq \hat{t}_{j}^{\prime}+2 \sigma$. Here again, any time-stamped label $\left(E, \hat{t}_{l}\right) \in D S_{1}$ cannot be assigned twice. Finally, it can be stressed that the output sequences of the 4-tuples of $M S_{1}$ and $M S_{2}$ will not be exactly the same as the incoming time-stamped label sequences and will also include their own missed detections and false alarms.

\section{Level 3: Fusion}

At this level, the sequences $M S_{1}$ and $M S_{2}$ are fused together to give two output sequences of time-stamped labels, $F S_{1}$ and $F S_{2}$, with the former related to the ECG channel and the latter to the SAP channel. First, redundancy in information that may exist between $M S_{1}$ and $M S_{2}$ is filtered to avoid twice processing 4-tuples present in both sequences. Then, the remaining 4-tuples, $\left(X, \hat{t}_{i}, Y, \hat{t}_{j}^{\prime}\right)$, where $(X, Y) \in\{(E, S),(E, \varnothing),(\varnothing, S)\}$, are fused sequentially and separately. Due to the Boolean character of labels $X$ and $Y$, two logical fusion operators have been alternately used for this purpose, namely, the AND and OR logic operators. For both, matches $\left(X, \hat{t}_{i}, Y, \hat{t}_{j}^{\prime}\right)$ such that $(X, Y) \equiv(E, S)$ are considered as nonconflicting and are, as a matter of fact, equally processed. Each match, $\left(E, \hat{t}_{i}, S, \hat{t}_{j}^{\prime}\right)$, is split by the fusion node, $F$, into a pair of time-stamped labels, $\left(E, \hat{t}_{i}\right)$ and $\left(S, \hat{t}_{j}^{\prime}\right)$, that are added, respectively, in the output sequences $F S_{1}$ and $F S_{2}$. But 
when dealing with conflicting matches, $\left(X, \hat{t}_{i}, Y, \hat{t}_{j}^{\prime}\right)$, for which $(X, Y) \equiv(E, \varnothing)$ or $(X, Y) \equiv(\varnothing, S)$, the AND and OR fusion operators act differently, as depicted in Tables 1 and 2. When acting as an AND, conflicting matches are simply disregarded by the fusion node, $F$, which means that all decisions, $\left(E, \hat{t}_{i}\right)$ and $\left(S, \hat{t}_{j}^{\prime}\right)$, taken at the first level that remain unmatched at the output of the second level, are a posteriori invalidated by $F$, which rather concludes the presence of artifacts at these times. But when acting as the logical operator OR, such decisions are not only validated by $F$, but true ventricular events are also inferred in $F S_{1}$ and $F S_{2}$ at times $\hat{t}_{j}^{\prime}-\hat{\bar{\Delta}}$ and $\hat{t}_{i}+\hat{\bar{\Delta}}$, respectively.

\section{Results}

Data Selection and Labeling

The proposed architecture has been tested on a noisy substract of the IMPROVE DL. We have chosen patient 2 as an example of someone who presented periods of unrest and variations of VR. As depicted Fig. 3, the noisy period includes parts of the ECG and SAP records in which there are, among others, a) total sensor failure in ECG as well as in SAP, and as a consequence, complete loss of signal; b) motion artifacts; and c) slow and abrupt baseline drifts. Other causes of noise in the data emanate from clinical interventions from nurse observations and care processes. For the creation of our noisy database, we have reassembled ECG and SAP signals that represent three hours of patient recordings, which correspond also to $\mathrm{NV}=$ 16650 successive VCs to be detected.

Hand-annotation was performed by a clinician for both channels. The time series $T S_{1}$ and $T S_{2}$ were defined so that the time occurrences of the true events coincide, respectively, with the peaks of the QRS complexes and with the peaks of the systolic pressure waves for the SAP channel. Due to random sensor failure, or instrumentation saturation, about 1000 QRS ventricular complexes and 500 systolic pressure waves can be truly considered as undetectable events by any one-dimensional signal-processing method.

\section{Level 1: Detection}

Performances of the three $\mathrm{VC}$ detectors were compared with each other from the traditional receiving operating characteristic (ROC) curves. As recommended by the Association for the Advancement of Medical Instrumentation (AAMI) [16], a beat-by-beat matching within $150 \mathrm{~ms}$ between beat labels from the annotated database used and those produced by the algorithm under test was required. Results are reported in Fig. 4. When focusing on the QRS-complex-detection ROC curves, no best QRS complex detector can be declared. Both provide: a) a very good detection probability (Pd close to 0.9 ) for a false-alarm rate greater than $10^{-2}$, b) a significant loss of performance (Pd less than 0.1) for a false-alarm rate less than $10^{-3}$. Between these two false-alarm rates, a rapid decrease in the detection probability can be observed for both detectors, which indicates a rather good bipolar behavior in the amplitude distribution of the corresponding decision statistics. Under these conditions, the QRS complex detector of Pan and Tompkins was retained.

A similar decrease is observed in the SAP detector ROC curve, but less abrupt, and for a false-alarm rate ranging from $10^{-2}$ to $10^{-4}$. This better result does not imply a better ability for the length transformation of Gritzali to detect $\mathrm{VC}$, but rather a more robust nature of the SAP compared with

\begin{tabular}{|c|c|c|}
\hline \multicolumn{3}{|c|}{$\begin{array}{c}\text { Table 2. Definition of the fusion rules } \\
\text { used for a fusion node } F \\
\text { acting as a logical operator OR. }\end{array}$} \\
\hline \multicolumn{3}{|c|}{$F \equiv O R$} \\
\hline Input & \multicolumn{2}{|c|}{ Output } \\
\hline$M S_{1} / M S_{2}$ & $F S_{1}$ & $F S_{2}$ \\
\hline$\left(E, \hat{t}_{i}, S, \hat{t}_{j}\right)$ & $\left(E, \hat{t}_{i}\right)$ & $\left(S, \hat{t}_{j}\right)$ \\
\hline$\left(E, \hat{t}_{i}, \varnothing, 0\right)$ & $\left(E, \hat{t}_{i}\right)$ & $\left(S, \hat{t_{i}}+\hat{\bar{\Delta}}\right)$ \\
\hline$\left(\varnothing, 0, S, \hat{t}_{j}\right)$ & $\left(E, \hat{t}_{j}^{\prime}-\hat{\bar{\Delta}}\right)$ & $\left(S, \hat{t}_{j}\right)$ \\
\hline
\end{tabular}

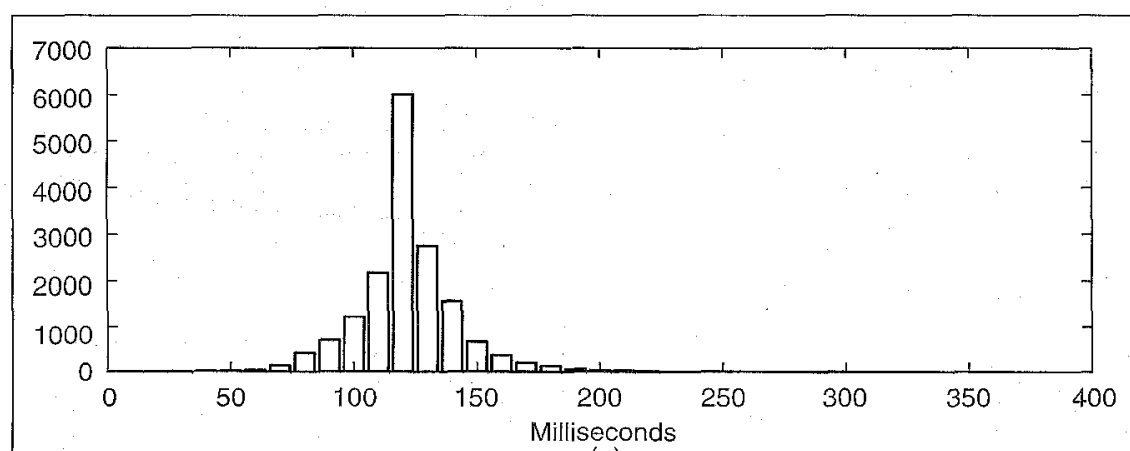

(a)

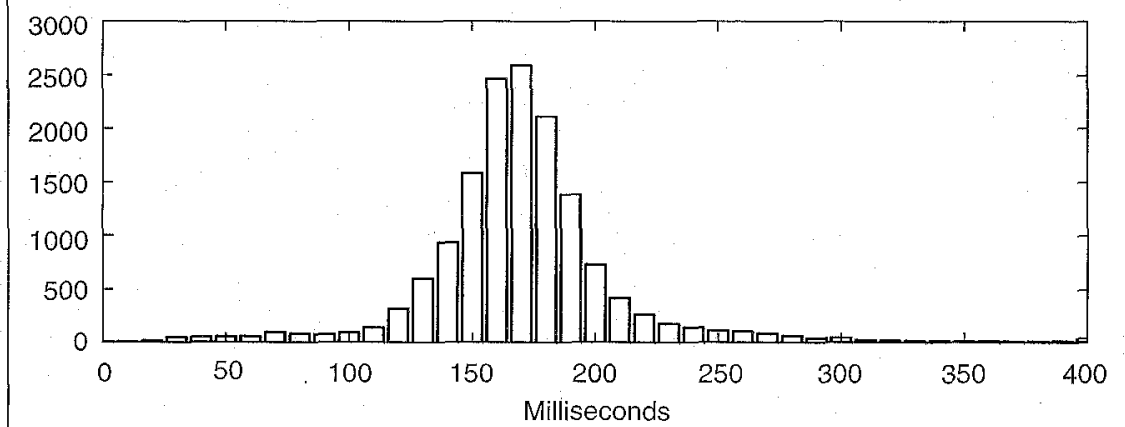

(b)

5. Estimated mean electromechanical delay histogram: a) when estimated with the hand-annotated noisy database, $b$ ) when estimated at the output of the $D_{1}^{P T}$ and $D_{2}^{G R}$ detectors, both tuned with a $10^{-2}$ false-alarm rate. 


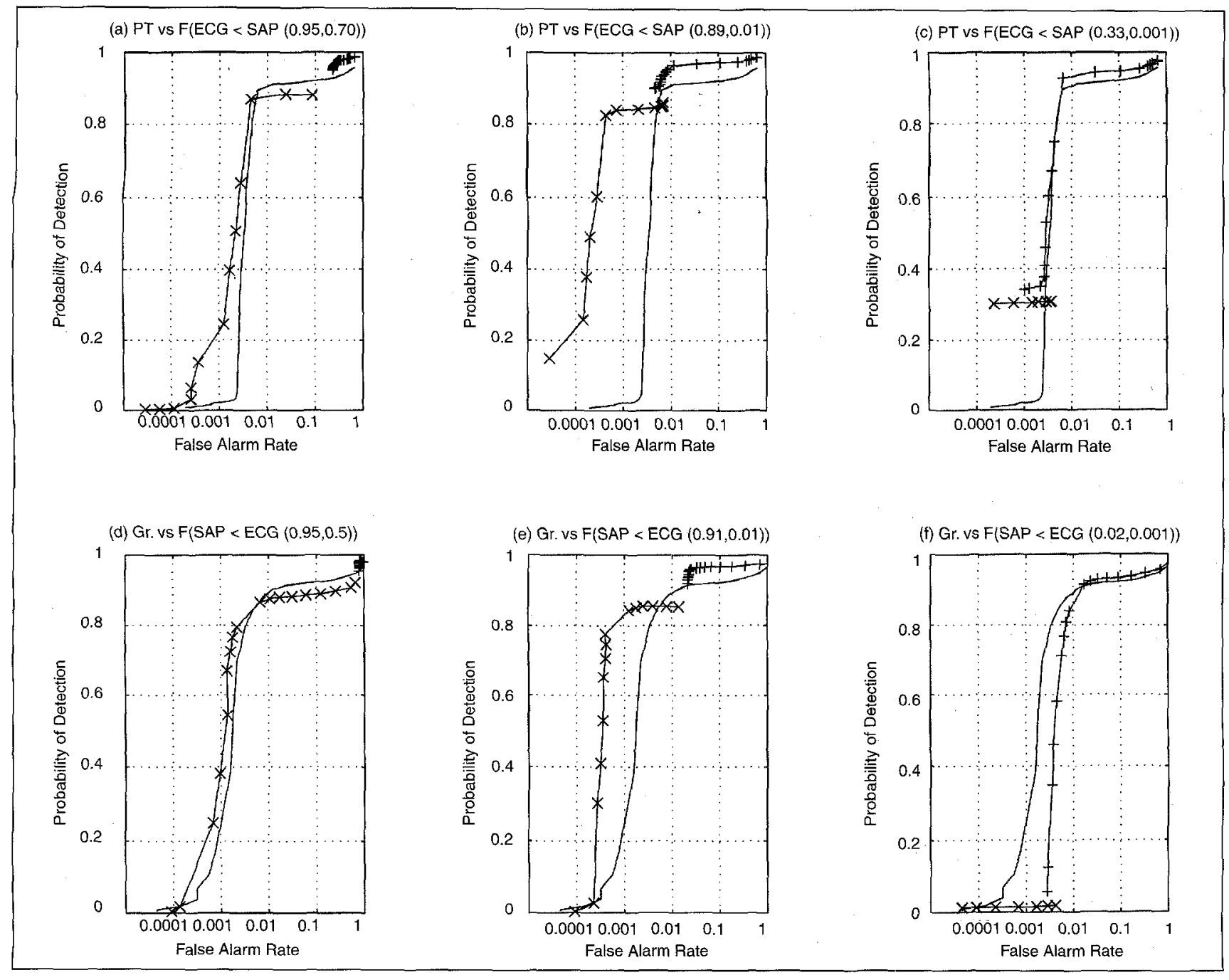

6. VC detection ROC curves obtained at the output of Level 3 (fusion): a, b, c) when compared with the VC detection ROC curve of Level 1, channel 1, for three fixed amounts of SAP ventricular rhythm information fused with ECG; and d, e, f) when compared with the VC detection ROC curve of Level 1, channel 2, for three fixed amounts of ECG ventricular rhythm information fused with SAP. ROC curves obtained with the fusion node, $F$, acting as the AND operator, are depicted with symbol "X,"while ROC curves depicted with the symbol " + " correspond to a fusion node, $F$, acting as the logic operator OR.

annotated noisy database, yielding the histogram depicted in Fig. 5(a), which confirms the initial value given to $\hat{\bar{\Delta}}$. But, when matching was carried out at the output of the detectors, both tuned to a common $10^{-2}$ false-alarm rate, a novel value of $170 \mathrm{~ms}$ was achieved for $\hat{\bar{\Delta}}$, as seen in Fig. 5(b). Moreover, an increase in the standard deviation can be observed, indicating the presence of a jitter phenomenon in the outcoming detection times from the first level. To compensate for this drawback, a large enough value of $100 \mathrm{~ms}$ was finally defined for $\sigma$, whereas a $145 \mathrm{~ms}$ value for $\hat{\bar{\Delta}}$ was retained as a compromise between 120 $\mathrm{ms}$ and $170 \mathrm{~ms}$. For the defined parameters $\sigma$ and $\hat{\bar{\Delta}}$, the $M S_{1}$ sequence resulted in $2.17 \%$ unmatched ECG detection points, versus $8.45 \%$ unmatched SAP detection points in $M S_{2}$.

\section{Level 3: Fusion}

Two kinds of experiments were conducted to evaluate the benefits of the proposed data-fusion approach in VR tracking, relative to the one-dimensional VC detectors used. First, VC detection performances were evaluated for each channel by means of ROC curves and for different amounts of VR information coming from the opposite channel. Figures 6(a-c) illustrate the QRS-complex-detection performances obtained for a decreasing amount of rhythm information coming from the SAP channel. Figures 6(d-f) correspond to the reverse case, where the ECG is considered this time as extra information for SAP event detection in the data-fusion scheme. ECG and SAP detector ROC curves have been reported, respectively, in Fig. 6(a-c) and $6(d-f)$ for comparison purposes. Whichever case is considered, results obtained for the OR fusion rule show that the false-alarm rate remains, to a first approximation, lower bounded by the highest false-alarm rate present at the input of the fusion node. Conversely, for the AND logical fusion operator, the output false-alarm rate can be considered as upper bounded by the lowest input false-alarm rate. Compared with the ROC curves of both one-dimensional VC detectors used, data fusion yields to better results: a) for false-alarm rates less than $5.10^{-3}$-except for Fig. 6(f), where 


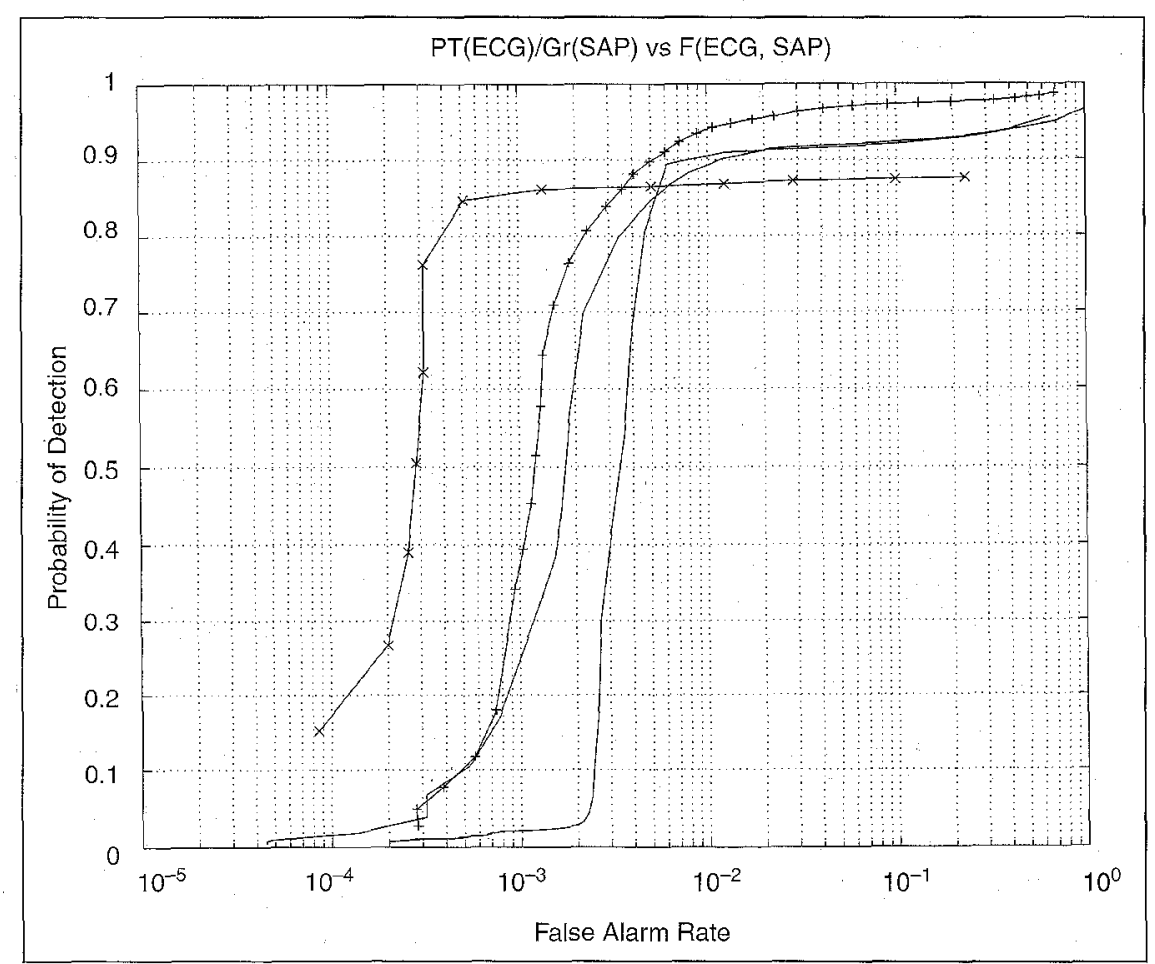

7. VC detection ROC curves obtained at the output of Level 3 (fusion), for a fusion node, $F$, acting as an AND ("X"), or as an OR (""), logical operator, and for frontend detectors, $D_{1}^{P T}$ and $D_{2}^{G R}$, both tuned with successive common false-alarm rates.

the ECG additional rhythm information remains inadequate, with a fusion node acting as an AND; and b) for the greatest false-alarm rates, but with a fusion node acting as an $O R$. In this experiment, best results were obtained for a $10^{-2}$ false-alarm rate, as reported Fig. 6(b) and $6(\mathrm{e})$, whether or not extra rhythm information comes from ECG or SAP.

A second experiment, classically used in distributed detection, consists in evaluating the data-fusion scheme when front-end detectors have been tuned with common false-alarm rates. Data-fusion results obtained in this configuration are shown Fig. 7 and compared with the ROC curves of each VC detector. An increase in the VC detection performance is always observed for the OR fusion rule, whereas the AND fusion operator makes the VC detector, based on data fusion, optimal for false-alarm rates less than $2.10^{-2}$.

\section{Conclusion}

A novel approach for robust cardiac VR tracking, based on electrophysiologic and haemodynamic data fusion, has been described. Evaluation, performed on a short but noisy subset of the IMPROVE DL, shows better VC detection perform- ances for the data-fusion approach than for the two other one-dimensional QRS detectors used, namely, the one proposed by Pan and Tompkins, and the length transformation of Gritzali. We are aware that the proposed data-fusion approach needs to be assessed on a larger database, such as the IMPROVE DL, as compared with a 2-D ECG detector, such as the one proposed by Gritzali [5]. But it must be emphasized that the proposed approach can easily be extended to the processing of an $N$-dimensional input signal, which can be either monomodal ( $N$-lead ECG) or multimodal (ECG, invasive/noninvasive blood pressure signals, phonocardiogrammas, etc.), provided information related to the ventricular activity is available on each channel. Multichannel matching as well as fusion between ventricular events will be accordingly adapted, but the ability of this approach to deal with asynchronous detections remains unchanged. Other fusion operators such as the MAJORITY, or M among N, could be envisaged in this case. Finally, the proposed methodology remains valid for multichannel tracking of events other than the QRS complex, for example, the $\mathrm{P}$ wave, from a pair of surface and oesophageal ECG signals.

\section{Acknowledgments}

The authors would like to thank Dr. Cliff Morgan from the Royal Brompton Hospital, London, for his advice during this current work, as well as Dr. Lotfi Senhadji, from the Laboratoire Traitement du Signal et de 1'Image, Rennes, for his helpful comments.

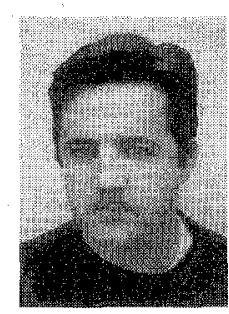

Laurent Thoraval was born in France on January 13,1966 . In 1995, he received the Doctorat degree in signal processing and telecommunications from the Universite de Rennes I, France. He is presently working at the Lahoratoire Traitement du Signal et de I'Image, Université de Rennes I. His research interests include Markov modeling of ECG for automatic recognition of arrhythmia. His current work is focused on cardiovascular data fusion in ICU.

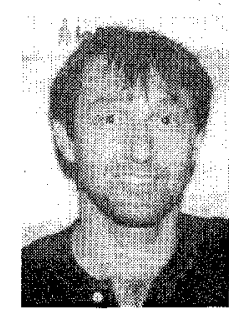

Guy Carrault received the Doctorat degree in signal processing and telecommunications from the Université de Rennes I, France. Since 1984, he has been working at the Laboratoire Traitement du Signal et de l'Image, Université de Rennes I. He is also a lecturer at the Institute Universitaire de Technologie de Rennes. His main research interests are in biomedical signal processing, more precisely ECG and EEG; concerned transients detection; automatic trends analysis; and segmentation of stationary signals. His current work is focused on cardiovascular data fusion in ICU.

Jean Marc Schleich received the doctorat degree in medicine from the University of Rennes in 1990 and a "Diplome d'étude approfondie" in the biomedical area in 1993. He is currently a clinician at the cardiovascular center of the University Hospital of Rennes. He is also a member of the Societé Française de Cardiologie. His research interests include cardiovascular data fusion in intensive coronary care unit.

Ron Summers was born in Billingham, Cleveland, UK, in 1957. He is currently a Senior Lecturer in Systems Science at City University, London, UK. He obtained a 
B.Sc. in Biophysical Science at the North East London Polytechnic (now University of East London) in 1982. His Masters degree in Information Engineering was taken in 1985 at City University, where his dissertation focused on the development of an expert system for the diagnosis of jaundice. Always interested in matters biomedical, he completed his Ph.D. in 1992 for which the theme was the design, implementation, and evaluation of an intelligent knowledge-based system for management of patients who required ventilatory therapy. He is an active member of both IMEKO and IFAC international committees and is committed to the development and use of medical informatics as a provider for enhanced patient care.

Maarten van de Velde is a Research Associate at the Eindhoven University of Technology, Medical Electrical Engineering group. He graduated there analyzing the spectral features of electroencephalographic signals during anesthesia. He has been working on signal-processing software for the group's research on evoked potentials and was part of the development team for standardized data acquisition in an international clinical study. His Ph.D. research investigates methods for validation of brain potentials.
Address for Correspondence: M. Laurent Thoraval, Laboratoire Traitement du Signal et de l'Image, Campus de Beaulieu, BT 22, Université de Rennes I, 35042 Rennes Cedex, France. E-mail : thoraval@Itsi.univ-rennes1.fr

\section{References}

1. Schleich JM: La caractérisation d'arythmies en unité de soins intensifs pour coronariens par approche fusion de données multisources/multicapteurs, DEA Signaux et Images en Biologie et Médecine, Universités du Grand Ouest, 1993.

2. Thoraval L: Analyse statistique de signaux électrocardiographiques par modèles de Markov cachés, Thèse de 1'Université de Rennes I, 1995. 3. Russel RO, Rackleyc E: Haemodynamic monitoring in a coronary intensive care unit, $\mathrm{Fu}-$ tura Publishing Company, 1981.

4. Pipberger HV: Computer analysis of the Electrocardiogram, In Stracy RW, Ed Waxman B., Computers in Biomedical Research, Vol. 1, New York, Academic, 1965

5. Gritzali F: Towards a generalized scheme for QRS detection in ECG waveforms, Signal Processing, Vol. 15, 1988, pp. 183-92.

6. Ligtenberg A: Reconnaissance de formes appliquée aux signaux Electrocardiographiques en vue d'une surveillance automatique, Ecole Polytechnique Federale de Lausenne, PhD, 1984.

7. Nieminen K, Langford RM, Morgan CJ, D'Hollander A, Kari A: A Clinical Description of the IMPROVE Data Library, IEEE Eng. Medicine and Biology, 16(6), pp.21-24, 401997.

8. Tenney RR, Sandell NR: Detection with distributed sensors, IEEE Trans. Aerospace Elec- tronic systems, Vol. AES-17, No. 4, 1981, pp. 501- 10 .

9. Boyer MO, Picinbono B: Quantization and Distributed Detection, Conf IEEE-ASSP, 1989, pp. 2688-91.

10. Chair Z, Sandell NR: Optimal data fusion in multiple sensor detection systems, IEEE Trans. Aerospace Electronic systems, Vol. AES-22, No. 1, 1986, pp. 98-101.

11. Krzysztofowicz R, Long D: Fusion of detection probabilities and comparison of multisensor systems, IEEE Transactions on Systems, Man, and Cybernetics, Vol. 20, No. 3, 1990, pp. 665-77.

12. Reibman AR, Nolte LW: Optimal detection and performance of distributed sensor system, IEEE Trans. Aerospace Electronic systems, Vol. AES-23, No. 1, 1987, pp. 24-30.

13. Thomopoulos SCA, Viswanathan $R$, Bougoulias 1D: Optimal decision fusion in Multiple sensor systems, IEEE Trans. Aerospace Electronic systems, Vol. AES-23, No. 5, 1987, pp. 644-53.

14. Chang W, Kam M: Asynchronous distributed detection, Proc. of 31 th conference on décision and control, December 1992, pp. 3498-503.

15. Pan J, Tompkins WJ: A real-time QRS detection algorithm, IEEE Trans. Biomedical Engineering, Vol. BME-32, 1985, pp. 230-36.

16. Association for the Advancement of Medical Instrumentation (AAMI). : Recommended practice for testing and reporting results of ventricular arrhythnnia detection algorithms, Tech. Rep. AAMIECAR-1987, AAMI, Arlington, VA, 1987.

17. Weissler AM, Lewis RP, Leighton RF: the systolic time intervals as a measure of left ventricular performance in man, Progression in cardiology, Eds PH Yu, MD and JF Goodwin, MD. Lea Febeger, Philadelphia, 1972. 